\title{
Excited state dynamics of some nonsteroidal anti-inflammatory drugs: a surface-hopping investigation.
}

\author{
Neus Aguilera-Porta ${ }^{\mathrm{a}, \mathrm{c}, \mathrm{d}}$, Inés Corral ${ }^{\mathrm{a}, \mathrm{b}, *}$, Jordi Munoz-Muriedas ${ }^{\mathrm{c}}$, Giovanni Granucci ${ }^{\mathrm{d}, *}$ \\ ${ }^{a}$ Departamento de Química, Universidad Autónoma de Madrid, 28049 Cantoblanco, Madrid, Spain \\ ${ }^{b}$ IADCHEM. Institute for Advanced Research in Chemistry, Universidad Autónoma de Madrid, 28049 Cantoblanco, Madrid, \\ Spain \\ ${ }^{c}$ Computational Toxicology, GlaxoSmithKline, Ware, Hertfordshire, UK \\ ${ }^{d}$ Dipartimento di Chimica e Chimica Industriale, Università di Pisa, via Moruzzi 13, 56124 Pisa, Italy
}

\begin{abstract}
Here we present a computational investigation of the excited state dynamics of 5 different active medical substances (aspirin, ibuprofen, carprofen, suprofen, indomethacin) which belong to the family of nonsteroidal anti-inflammatory drugs (NSAIDs). The nonadiabatic dynamics simulations were performed using the surface hopping method, with electronic energies and couplings evaluated on the fly in a semiempirical framework. For aspirin, the solvent decay dynamics was also considered by inserting it in a cluster of water molecules, following a QM/MM scheme. A quite diverse behavior was observed for the systems considered, going from fast deactivation to the $S_{0}$ (aspirin and ibuprofen), to ultrafast intersystem crossing to the triplet manifold (carprofen and suprofen), or to the generation of long lived $S_{1}$ states (indomethacin). To our knowledge, this is the first computational study of the photodynamics of these NSAIDs.
\end{abstract}

Keywords: NSAIDs, Excited state dynamics, Intersystem crossing, Triplet quantum yields, Surface hopping

\section{Introduction}

Drugs are exposed to natural or artificial light along the pharmaceutical chain, from their manufacture until their dispensation or even after administration. The interaction between a drug and ultraviolet (UV) light may give rise to phototoxic and/or photoallergic side effects. Both kind of processes are referred to as "photosensitivity" [1, 2]. A very well known family of drugs which may give rise to photosensitive reactions is that of nonsteroidal anti-inflammatory drugs (NSAIDs), which are used mainly as analgesic, antipyretic and anti-inflammatory agents [1, 2].

Photosensitive reactions are complex processes, involving different pathways such as energy, electron or hydrogen transfer, photodecomposition and photobinding. The investigation of the photophysics of drugs or the photoreactive paths that might be activated upon photon absorption is crucial for identifying photoinitiated side effects, as well as in the design of novel and improved drugs. In the literature, there are a few experimental and computational studies on the photophysics of NSAIDs, most often based on steady state results [3, 4, 5, 6, 7, 8, 9, 10, 11, 12, 13, 14], or on quantum chemical calculations on the energetics of their excited states, mainly at DFT/TD-DFT level of theory [2, 15, 16, 17, 18]. However, investigations of the excited state dynamics of these molecules are still lacking.

In the present work, we considered five NSAIDs: aspirin (ASP), ibuprofen (IBU), carprofen (CAR), suprofen (SUP) and indomethacin (IDM), see Figure 1. Our aim is the study of their excited state dynamics

\footnotetext{
* Corresponding author

Email addresses: neus.aguilera@uam.es (Neus Aguilera-Porta), ines.corral@uam.es (Inés Corral), jordi.4.munoz-muriedas@gsk.com (Jordi Munoz-Muriedas), giovanni.granucci@unipi.it (Giovanni Granucci)
} 


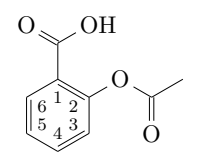

Aspirin (ASP)

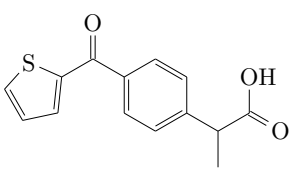

Suprofen (SUP)

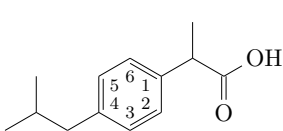

Ibuprofen (IBU)

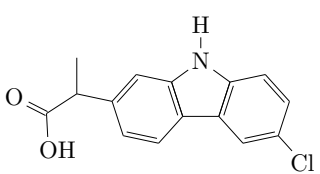

Carprofen (CAR)

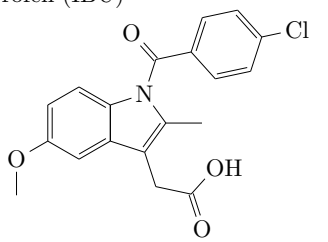

Indomethacin (IDM)

Figure 1: The nonsteroidal anti-inflammatory drugs considered in the present work.

through nonadiabatic molecular dynamics simulations, in order to gain information about their gas phase photophysics (singlet lifetimes, triplet quantum yields, and the related mechanisms and pathways). In the case of ASP, the effect of the solvent (water) along the relaxation dynamics has been simulated as well. We believe that our results will be also helpful in the selection of descriptors for quantitative structure-activity relationships (QSAR) models [19] that could be used to predict photoactivity of new or untested derivatives of these NSAIDs.

The outline of this paper is as follows. Section 2 introduces the computational strategy adopted for the on the fly molecular dynamics simulations (a methodological improvement is reported in the appendix), section 3 describes the excited states dynamics, and concluding remarks are offered in section 4.

\section{Method}

For the on the fly nonadiabatic dynamics simulations of the molecular systems shown in Figure1, potential energy surfaces (PES) and couplings were obtained with the Floating Occupation Molecular OrbitalsConfigurations Interaction (FOMO-CI) method [20, 21], using the semiempirical AM1 Hamiltonian with standard parameterization. We show in Table 1 the active spaces used in the FOMO-CI calculations for the systems considered in this work. In all the cases, the CI space was of CAS type. For all the molecular systems considered, the orbitals included in the FOMO-CI active space can be classified as $\pi$ and $\pi^{*}$, at least in the vicinity of the ground state equilibrium geometry (see Figure S1 of the Supporting Information). For those systems for which no TD-DFT absorption spectra have been reported in the literature, i.e. Carprofen and Indomethacin, we have computed the energies of the first transitions using the TD-CAMB3LYP/6$31+\mathrm{G}(\mathrm{d}, \mathrm{p})$ protocol at the ground state geometries optimized with the same functional and basis set, that will serve to assess the reliability of the AM1 method in the prediction of the electronic energies for these systems. The most important configurations contributing to each electronic state and the orbitals involved in these configurations can be found in Table S1 and Figures S2 and S3 of the Supporting Information.

Spin-orbit (SO) couplings, needed for the description of intersystem crossing processes in the dynamics simulations, were evaluated with a mean field Hamiltonian [22]. The relevant semiempirical parameters for $\mathrm{C}, \mathrm{O}, \mathrm{Cl}$ and $\mathrm{N}$ atoms were obtained [22] from the splitting of the ground state atomic terms (for $\mathrm{N}$ the ${ }^{2} D^{\circ}$ term was considered, as the ground state ${ }^{4} S^{\circ}$ has no splitting) and their respective values are 28.6, 170, 588 and $4 \mathrm{~cm}^{-1}$. For the $\mathrm{S}$ atom, we used the same semiempirical spin-orbit parameter $\left(500 \mathrm{~cm}^{-1}\right)$ employed in a previous work on thioguanine [23].

The molecular dynamics simulations were performed with the fewest switches surface hopping method [21, 24, 25]. The trajectories were propagated on the "spin-adiabatic" potential energy surfaces, obtained diagonalizing the electronic Hamiltonian (including the SO coupling) in the subspace spanned by the first few spin-defined electronic states (the "spin-diabatic" states, see Table 1) [23]. The integration of the electronic time dependent Schrödinger equation was performed with the widely used Local Diabatization (LD) scheme 
Table 1: CAS-CI active spaces (electrons/orbitals), number of spin-diabatic states (singlets, triplets) and number of trajectories considered in the dynamics simulations. "Non valid" trajectories were discarded. ASP $(a q)$ labels aspirin in a cluster of water molecules.

\begin{tabular}{lcccc}
\hline \hline molecule & active & $S, T$ & \multicolumn{2}{c}{ \# of trajectories } \\
\cline { 4 - 5 } & space & & total & Non Valid \\
\hline ASP $_{\mathrm{ASP}_{(a q)}}^{4 / 4}$ & $4 / 4$ & 3,4 & 401 & 0 \\
$\mathrm{IBU}$ & $4 / 3$ & 3,4 & 440 & 0 \\
$\mathrm{CAR}$ & $4 / 4$ & 3,4 & 203 & 20 \\
$\mathrm{SUP}$ & $6 / 5$ & 4,3 & 364 & 60 \\
$\mathrm{IDM}$ & $6 / 5$ & 3,3 & 304 & 9 \\
\hline \hline
\end{tabular}

Table 2: Semiempirical FOMO-CI vertical excitation energies (eV) and oscillator strengths (in parenthesis) computed at the ground state equilibrium structure. Experimental data for the excitation energies (in terms of maxima of the absorption spectra) are shown in italics.

\begin{tabular}{|c|c|c|c|c|c|}
\hline transition & $\overline{\mathrm{ASP}}$ & $\overline{\mathrm{IBU}}$ & CAR & $\overline{\text { SUP }}$ & $\overline{\mathrm{IDM}}$ \\
\hline$S_{0} \rightarrow S_{1}$ & $4.244 .13^{a}(0.014)$ & $5.124 .56^{c}(0.47)$ & $3.813 .76^{d}(0.03)$ & $4.504 .29^{e}(0.25)$ & $4.193 .8^{r f}(0.74)$ \\
\hline$S_{0} \rightarrow S_{2}$ & $4.634 .49^{b}(0.027)$ & $5.194 .68^{c}(0.36)$ & $3.984 .13^{d}(0.01)$ & $4.584 .63^{e}(0.59)$ & $4.234 .64^{f}(0.33)$ \\
\hline$S_{0} \rightarrow S_{3}$ & $5.875 .41^{b}(0.76)$ & $8.29(0.00)$ & $4.885 .17^{d}(1.52)$ & $5.11(0.39)$ & $4.985 .39^{f}(0.26)$ \\
\hline$S_{0} \rightarrow T_{1}$ & 3.16 & 3.24 & 3.29 & 2.70 & 2.98 \\
\hline$S_{0} \rightarrow T_{2}$ & 3.78 & 3.84 & 3.38 & 2.89 & 3.13 \\
\hline$S_{0} \rightarrow T_{3}$ & 3.86 & 8.31 & 3.67 & 3.26 & 3.41 \\
\hline$S_{0} \rightarrow T_{4}$ & 4.48 & - & 3.97 & 5.81 & 5.59 \\
\hline
\end{tabular}

Absorption spectrum in DMSO [15], shoulder. ${ }^{b}$ Absorption spectrum in various solvents [3, 15, 33]. $c$ Absorption spectrum in water [5]. ${ }^{d}$ Absorption spectrum in ethanol [6]. $e$ Absorption spectrum in acetonitrile [8]. ${ }^{f}$ Absorption spectrum in water [12, 9].

[25], which is very convenient in the case of weakly avoided crossings [26] [27]. In the present work we used a new algorithm for the evaluation of the fewest switches transition probability, described in the Appendix, especially useful in the case of many weakly interacting states (consider that each triplet state is split in three components by the SO interaction). Quantum decoherence was approximately taken into account with the ODC method [28]. The time step used in the propagation of the trajectories was $\Delta t=0.1 \mathrm{fs}$.

ASP-solvent interaction has been described within a QM/MM framework with electrostatic embedding: the aspirin molecule was inserted in a spherical cluster of 775 water molecules, described with a TIP3P force field. This system will be labeled $\mathrm{ASP}_{(a q)}$ from now on. To avoid evaporation of water molecules from the surface of the cluster during the dynamics simulations, a constraining potential was added [29].

The starting conditions for the surface hopping calculations were sampled from thermal equilibrated trajectories, propagated on the ground state potential energy surface for $50 \mathrm{ps}\left(10 \mathrm{ps}\right.$ for $\left.\operatorname{ASP}_{(a q)}\right)$ at $300 \mathrm{~K}$ with the Bussi-Parrinello thermostat [30]. Each surface hopping trajectory starts with a vertical excitation to a state selected taking into account the radiative transition probability. The sampling procedure is described in detail in ref. 21]. The number of total trajectories obtained for each system is shown in Table 1. together with the number of trajectories discarded for technical reasons that, thus, were excluded from the analysis. The surface hopping trajectories were propagated for $10 \mathrm{ps}$. In discussing the results of the dynamics simulations, we shall call "state population" to the average population of the spin-diabatic states, evaluated as shown in ref. [23]. All the molecular dynamics simulations were carried out with a developing version of the MOPAC2002 [31] package, interfaced to the TINKER 32] Molecular Mechanics package in the $\mathrm{QM} / \mathrm{MM}$ calculations. 

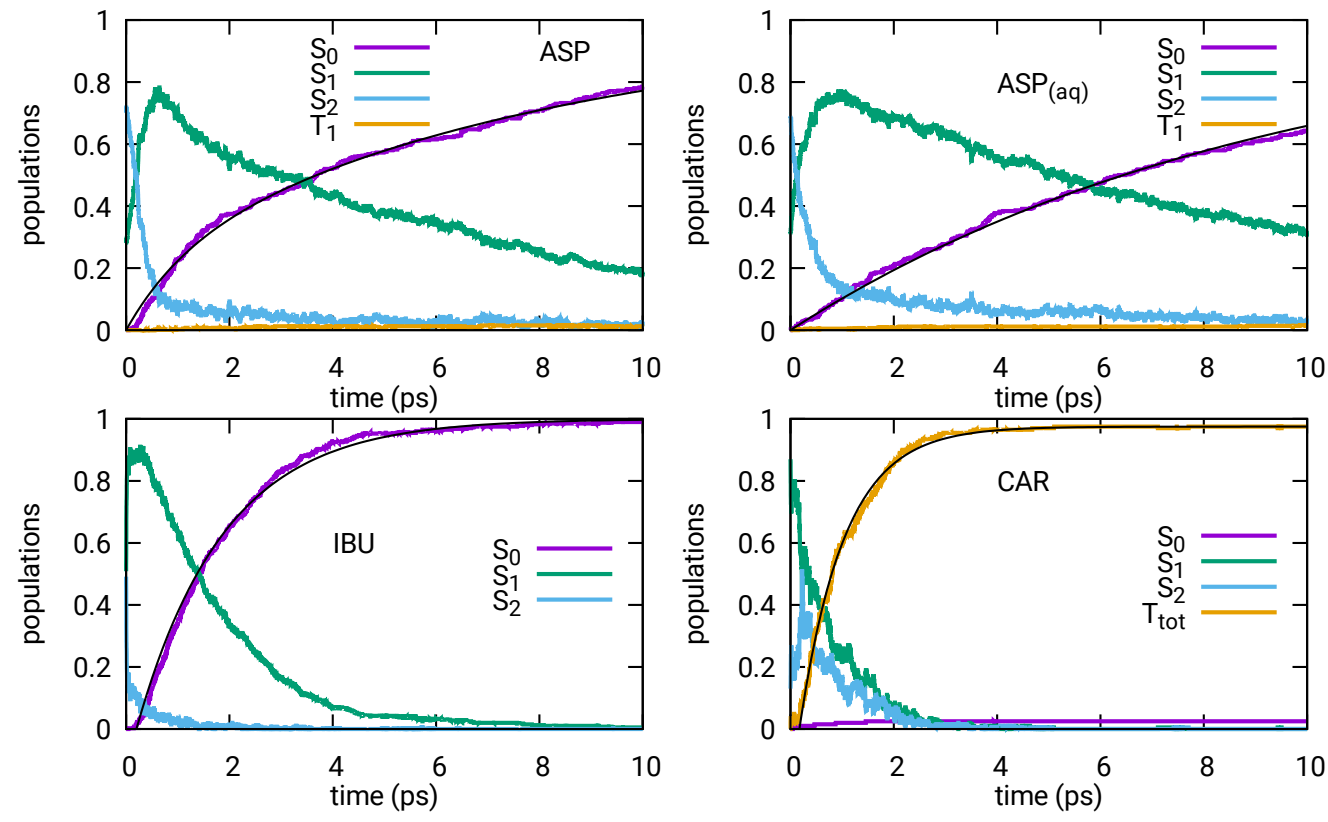

Figure 2: Time evolution of the population of the spin-diabatic states for aspirin (in vacuum and in a cluster of water molecules), ibuprofen and carprofen. For carprofen $T_{t o t}$ labels the total triplet population. Black curves are fits of the $S_{0}$ or $T_{\text {tot }}$ populations.

\section{Results and discussion}

\subsection{Aspirin $(A S P)$}

Our computed semiempirical FOMO-CI vertical excitation energies for ASP in vacuum, reported in Table 2. match quite well the experimental results, especially if the shoulder at about $300 \mathrm{~nm}(4.13 \mathrm{eV})$ of the peak at $276 \mathrm{~nm}(4.49 \mathrm{eV})$ in the spectrum obtained by Govindasamy et al. [15] in DMSO can be identified with the $S_{0} \rightarrow S_{1}$ transition. Other experimental absorption spectra of ASP recorded in various solvents [3, 15, 33] exhibit also maxima at $276 \mathrm{~nm}(4.49 \mathrm{eV})$ and $229 \mathrm{~nm}(5.41 \mathrm{eV})$. Although red-shifted, our AM1 vertical transitions are also in reasonable agreement with the B3LYP $/ 6-311 \mathrm{G}^{* *}$ values reported in [16], the second absorption being the most sensitive to the level of theory employed in the calculations with a deviation amounting to ca. $0.75 \mathrm{eV}$. At the Franck-Condon region, the first two excited singlets, $S_{1}$ and $S_{2}$, both have mixed character $H \rightarrow L, H-1 \rightarrow L, H \rightarrow L+1$ and $H-1 \rightarrow L+1$, with the $H \rightarrow L$ configuration having the largest weight for $S_{1}$, according to the AM1 FOMO-CI calculations.

In Figure 2 we show the time evolution of the population of the spin-diabatic states of ASP (top left panel). Our surface hopping trajectories start with excitations to $S_{1}$ or $S_{2}$, in agreement with the oscillator strengths of Table 2. At the beginning, the $S_{2}$ state is the most populated. The corresponding excitation energy, averaged over the full swarm of 401 trajectories, is $4.49 \mathrm{eV}$. The spin-orbit couplings evaluated at the $S_{0}$ equilibrium geometry for the first few electronic states were very weak (of the order of $1 \mathrm{~cm}^{-1}$, or less), for this and the other systems considered in this work. Within the first picosecond, the $S_{2}$ population drops from 0.72 to about 0.07 , due to $S_{2} \rightarrow S_{1}$ transitions. Actually, $S_{1}$ and $S_{2}$ are very close in energy, and keep exchanging population during the 10 ps of dynamics considered: the average number of $S_{2} \rightarrow S_{1}$ and $S_{1} \rightarrow S_{2}$ transitions per trajectory amounts respectively to 8.5 and 7.8 .

Within the first $10 \mathrm{ps}$, the triplet quantum yield is too low (0.015) to be statistically meaningful: only 6 trajectories out of 401 perform a transition from $S_{1}$ to $T_{1}$. Hence, the decay from $S_{1}$ is almost exclusively represented by the internal conversion to $S_{0}$, which happens either through a $S_{1} / S_{0}$ conical intersection 


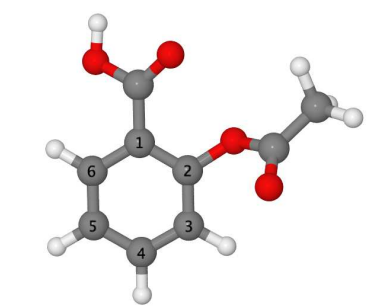

$S_{0}$ mimimum $(0 \mathrm{eV})$

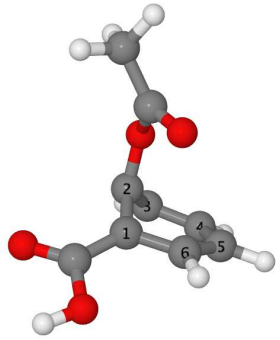

$S_{0} / S_{1} \mathrm{Cl} \quad(3.96 \mathrm{eV})$ puckering at $\mathrm{C}_{2}$

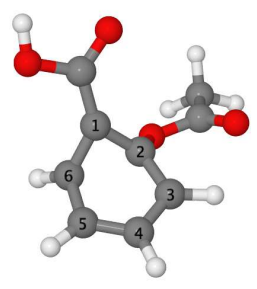

$S_{0} / S_{1} \mathrm{Cl}(5.01 \mathrm{eV})$
puckering at $\mathrm{C}_{1}$

Figure 3: Aspirin. Presented are the AM1 FOMO-CI minimum energy geometries of the ground state, of the $S_{1} / S_{0}$ seam with puckering at $\mathrm{C}_{2}$, and of the $S_{1} / S_{0}$ seam with puckering at $\mathrm{C}_{1}$.

(40\% of cases), or at large energy difference between the two states (60\%). We found in ASP a minimum energy CI (conical intersection) lying at $3.96 \mathrm{eV}$ above the ground state minimum and corresponding to the puckering of the aromatic ring at the ring carbon atom $\mathrm{C}_{2}$ (see Figure 3), linked to the acetoxy group. That crossing seam is important in the dynamics. In fact, all the trajectories making a $S_{1} \rightarrow S_{0}$ transition with an energy difference between the two states $E\left(S_{1}-S_{0}\right)<1.5 \mathrm{eV}$, show evident puckering of the aromatic ring at $\mathrm{C}_{2}$. Another $S_{1} / S_{0}$ minimum energy CI, corresponding to puckering of the aromatic ring at $\mathrm{C}_{1}$, is found at $5.01 \mathrm{eV}$ above the $S_{0}$ minimum, and does not play any role in the dynamics (see Figure 3).

The $S_{1}$ state of ASP has a "planar" minimum (i.e. with a planar conformation of the aromatic ring) at $4.12 \mathrm{eV}$ above the ground state equilibrium energy, easily accessible from the Frank-Condon region. Puckering of the aromatic ring at $\mathrm{C}_{2}$ would lead to the $S_{1} / S_{0}$ CI referred above, which actually lies $0.16 \mathrm{eV}$ below the planar minimum of $S_{1}$. However, such a pathway does not seem to be easily accessed during the dynamics, considering that $60 \%$ of the trajectories in $S_{1}$ stay trapped in the planar minimum, eventually hopping to $S_{0}$ from there, with a large $S_{1}-S_{0}$ energy difference (3.97 eV in average).

The rise of the $S_{0}$ population is well reproduced by the biexponential function

$$
P_{S_{0}}(t)=1-w e^{-t / \tau_{1}}-(1-w) e^{-t / \tau_{2}}
$$

with $w=0.23, \tau_{1}=1.13 \mathrm{ps}$ and $\tau_{2}=8.21 \mathrm{ps}$. At early times (for $t<2 \mathrm{ps)} \mathrm{the} S_{1} \rightarrow S_{0}$ internal conversion through the $S_{1} / S_{0}$ CI dominates with respect to the transitions from the $S_{1}$ planar minimum, so that the shorter lifetime $\tau_{1}$ could be mainly ascribed to the decay via conical intersection. The situation is reversed at later times. Notice that the $S_{2}$ population drops below 0.1 after just $600 \mathrm{fs}$, when the population of $S_{0}$ is still below 0.13 . Therefore, the rise of $P_{S_{0}}$ is not expected to be much influenced by the $S_{2} \rightarrow S_{1}$ decay rate. It is important to note here that slow decays from weak coupling regions are especially difficult to be accurately reproduced with surface hopping approaches [28]. We expect such kind of inaccuracies inherent to the method to moderately affect the value estimated for $\tau_{2}$.

We now switch to $\operatorname{ASP}_{(a q)}$ (i.e. ASP inserted in a spherical cluster of water molecules). As it can be appreciated from Figure 2] the decay pattern of $\mathrm{ASP}_{(a q)}$ is pretty much coincident with that of ASP in vacuum, with just a modest slowdown of transition rates, which can be ascribed to solvent friction. We notice that solvents are quite effective in slowing down the nonadiabatic transitions. In fact, vibronic coupling is proportional to the nuclear velocities, but the fastest nuclei, exploring a larger portion of their configurational space, are more subject to the interaction with solvent molecules. An appreciation of this effect can be obtained by monitoring in time the average nuclear kinetic energy $\left\langle E_{k i n}\right\rangle$ of the ASP molecule in different environments. Comparing $\left\langle E_{k i n}\right\rangle$ for ASP in vacuum and in the solvent cluster, we note that they start to diverge after just about $300 \mathrm{fs}$. By fitting $\left\langle E_{k i n}\right\rangle$ of ASP in the solvent cluster as in Ref. 34] we can also estimate the time constant for the vibrational cooling of ASP in water solution, which is found to be $30.8 \mathrm{ps}$.

A closer inspection of the excited states decay dynamics of $\mathrm{ASP}_{(a q)}$ reveals a striking difference with 


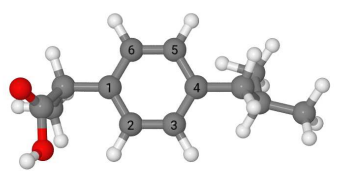

$S_{0}$ mimimum $(0 \mathrm{eV})$
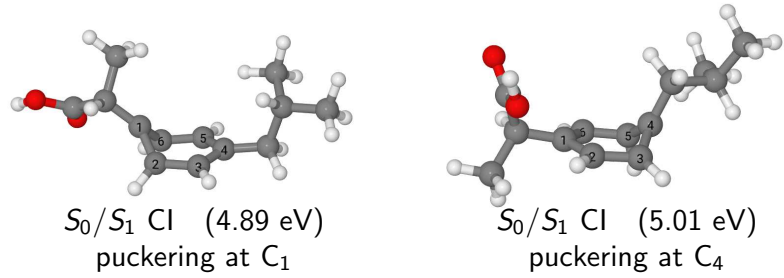

Figure 4: Ibuprofen. Presented are the AM1 FOMO-CI minimum energy geometries of the ground state, of the $S_{1} / S_{0}$ seam with puckering at $\mathrm{C}_{1}$, and of the $S_{1} / S_{0}$ seam with puckering at $\mathrm{C}_{4}$.

respect to the gas phase results (albeit expected to a certain extent). In particular, $S_{1} \rightarrow S_{0}$ internal conversion through the $S_{1} / S_{0}$ CI is almost completely suppressed. Thus, the transitions from the $S_{1}$ planar minimum represent $93 \%$ of the $S_{1} \rightarrow S_{0}$ decay. This effect might be ascribed to the steric interaction of the chromophore with the solvent cage. In fact, the puckering of the aromatic ring at $\mathrm{C}_{2}$ leads to a quite important geometrical change for the whole molecular system and its steric hindrance (as it can be appreciated by looking at Figure 3 , the $\mathrm{C}_{2}-\mathrm{O}$ bond is displaced from the plane of the ring to a position perpendicular to that plane). Hydrogen bonds with water molecules may also play a role in this respect, although they are rather established with the $\mathrm{COOH}$ group. In agreement with these findings, the rise of the ground state population can be fitted with a single exponential function, setting $w=0$ in equation 1 . and delivering then a value for $\tau_{2}=9.30 \mathrm{ps}$.

\subsection{Ibuprofen (IBU)}

The experimental UV spectrum of both neutral and deprotonated IBU in water solution, reported by Vione et al. [5], show a weak band $\left(\epsilon_{\max } \simeq 400 \mathrm{M}^{-1} \mathrm{~cm}^{-1}\right)$ with a couple of peaks at $265 \mathrm{~nm}(4.68 \mathrm{eV})$ and $272 \mathrm{~nm}(4.56 \mathrm{eV})$. Our vertical transition energies for $S_{2}$ and $S_{1}$ (see Table 22) are in reasonable agreement with the positions of the experimental peaks and calculated absorptions [18], both being about $0.5 \mathrm{eV}$ too high compared to the experimental results, but $0.1-0.4 \mathrm{eV}$ too low with respect to the calculated values, respectively. In this case, the $S_{1}$ and $T_{1}$ states have $H \rightarrow L$ character, while $S_{2}$ and $T_{2}$ have $H-1 \rightarrow L$ character (see Figure S1).

At the beginning of the dynamics $S_{1}$ and $S_{2}$ are populated, with an average excitation energy of $5.07 \mathrm{eV}$. The time evolution of the spin diabatic populations is qualitatively similar to ASP, and can be schematized as $S_{2} \rightleftharpoons S_{1} \rightarrow S_{0}$. However, both the decay from $S_{2}$ to $S_{1}$ and from $S_{1}$ to $S_{0}$ are faster for IBU. Concerning the $S_{2} \rightarrow S_{1}$ internal conversion process, the $S_{2}$ "planar" minimum (i.e. with a planar conformation of the aromatic ring, thus easily accessible from the Franck-Condon region) corresponds to a $S_{2} / S_{1}$ CI, at variance with ASP, which explains the ultrafast decay of the $S_{2}$ state for IBU.

The deactivation of $S_{1}$ towards $S_{0}$ involves several CI regions, that correspond to geometries where the aromatic ring is puckered. In particular, the most important $S_{1} / S_{0}$ conical intersections involve the puckering of the ring at the carbon atoms $\mathrm{C}_{1}$ and $\mathrm{C}_{4}$ (see Figure 4), and will be labeled in the following $\mathrm{CI}_{p}$ and $\mathrm{CI}_{a}$. These degeneracy points are, respectively, located $4.89 \mathrm{eV}$ and $5.01 \mathrm{eV}$ above the $S_{0}$ minimum. Very close to these intersections, we found a $S_{1}$ planar minimum which lies $4.97 \mathrm{eV}$ above the $S_{0}$ equilibrium geometry. About 55\% (30\%) of the $S_{1} \rightarrow S_{0}$ transitions go through the $\mathrm{CI}_{a}\left(\mathrm{CI}_{p}\right)$. The rest is conveyed through conical intersection regions which require the puckering at other carbon atoms of the ring, lying slightly higher in energy, in the range 5.03-5.15 eV. At variance with ASP, no slow decay from the $S_{1}$ planar minimum is found for IBU. As a result, the rise of the $S_{0}$ population can be fitted by a single exponential (see Figure 2), with a time constant of $1.89 \mathrm{ps}$.

It is known that IBU undergoes photolysis after UVB or UVC excitation in solution, for both the anionic deprotonated and the neutral forms [5, 35]. In a TD-DFT study, Musa and Eriksson [18] showed that the $\mathrm{T}_{1}$ state of deprotonated IBU has a very small barrier versus decarboxylation (only $0.3 \mathrm{Kcal} / \mathrm{mol}$ ). This is in agreement with our FOMO-CI calculations, where the $\mathrm{T}_{1}$ state of deprotonated IBU is found to be unstable with respect to decarboxylation. Vione et al. 5] found quite different photolysis quantum yields 
for neutral and deprotonated IBU (respectively, 1.0 and 0.3 ) suggesting that the neutral and deprotonated species would probably undergo dissociation via two different mechanisms. Our nonadiabatic dynamics simulations indicate that the photolysis of neutral IBU would occur from the hot ground state.

\subsection{Carprofen $(C A R)$}

At the ground state equilibrium geometry, the three rings of CAR have an almost planar conformation, with a very modest pyramidalization at the N atom, which disappears at $S_{1}$ and $S_{2}$ minima and becomes more pronounced at the $T_{1}$ equilibrium geometry. The $S_{1}, S_{2}$ and $T_{1}$ minima lie respectively at 3.71, 3.91 and $2.90 \mathrm{eV}$ above the ground state equilibrium energy. Consistently with the much larger difference between adiabatic (i.e. minimum-minimum) and vertical transition energies (see Table 2), $T_{1}$ shows a larger geometrical distortion compared to $S_{1}$ and $S_{2}$ from the Franck-Condon point. The experimental absorption spectrum [6] of CAR in ethanol shows a peak at $300 \mathrm{~nm}(4.13 \mathrm{eV})$ with a broad side band extending up to $350 \mathrm{~nm}$, and a more intense band at about $240 \mathrm{~nm}(5.17 \mathrm{eV})$. In the gas phase, TD-CAMB3LYP predict the first three transitions, all of $\pi \rightarrow \pi^{*}$ character, in the range between 4.3 and $5.3 \mathrm{eV}$, i.e $4.29,4.70$ and 5.30 eV, See Table S1 and Figure S2 of the Supporting Information. Our computed FOMO-CI vertical transitions $S_{0} \rightarrow S_{1}, S_{0} \rightarrow S_{2}$, and $S_{0} \rightarrow S_{3}$ are found at slightly lower energies with respect to the experimental data, see table 2. The fluorescence spectrum of CAR in ethanol [6] shows two peaks at $367 \mathrm{~nm}(3.38 \mathrm{eV})$ and $352 \mathrm{~nm}$ (3.52), which may be compared with our FOMO-CI vertical transition energies $S_{1} \rightarrow S_{0}(3.63 \mathrm{eV})$ and $S_{2} \rightarrow S_{0}(3.84 \mathrm{eV})$. The phosphorescence spectrum [6, 36] has a maximum at about $430 \mathrm{~nm}(2.88 \mathrm{eV})$; the corresponding calculated $T_{1} \rightarrow S_{0}$ vertical transition energy is $2.46 \mathrm{eV}$. Overall, the AM1 FOMO-CI energies show a reasonable agreement with the experimental data available.

In the simulations of CAR excited state dynamics, the starting populations of the $S_{1}$ and $S_{2}$ states are 0.13 and 0.87 , respectively, with an average excitation energy of $3.82 \mathrm{eV}$. The $S_{3}$ state, lying much higher in energy, was not included in the molecular dynamics simulations. Following excitation, the $S_{1}$ and $S_{2}$ population is transferred very rapidly to the triplet states $T_{1}-T_{4}$, which are very close in energy. The total triplet population rises with a time constant of $1.04 \mathrm{ps}$, and the triplet quantum yield after $10 \mathrm{ps}$ is 0.975 . All the triplets considered in the simulations receive population from the singlets, with about $80 \%$ of the transitions going to $T_{3}$ and $T_{4}$, the closest to $S_{1}$ and $S_{2}$. Once on the triplet manifold, the trajectories hop to $T_{1}$, so that the total triplet population is always almost coincident with that of $T_{1}$.

According to the experimental results of Bosca et al. [6], the triplet quantum yield of CAR in ethanol solution after excitation at $355 \mathrm{~nm}$ is 0.37 , and the fluorescence quantum yield is small but not negligible (0.06). Although our simulations correctly account for the decay to the triplet manifold, the population transfer to the triplets appears to be severely overestimated. It seems very unlikely that this can be attributed to an overestimation of the SO couplings, considering that our calculated values for the latter amount to 1 $\mathrm{cm}^{-1}$ or less. These discrepancies with the experimental results could be rather ascribed to inaccuracies in the description of the PES. However, the solvent may also play a role in slowing down the transition rate to triplet states.

\subsection{Suprofen (SUP)}

The experimental absorption spectrum of SUP in acetonitrile [8] (where the molecule is mainly present in its neutral form) shows two peaks at $264 \mathrm{~nm}(4.63 \mathrm{eV})$ and $289 \mathrm{~nm}(4.29 \mathrm{eV})$, which are red shifted upon deprotonation [8, 37]. Comparing these data with the calculated $S_{0} \rightarrow S_{1}$ and $S_{0} \rightarrow S_{2}$ vertical transition energies of Table 2, the $S_{1}$ and $S_{2}$ states appear to be too close in energy, in between the experimental peaks, still not far from them. By comparison with the experiment, the energy of the $S_{3}$ state is probably underestimated by our calculations. Our AM1 FOMO-CI calculations predict the first two $\pi \rightarrow \pi^{*}$ transitions in the same region of the spectrum as the B3LYP/6-31G(d,p) protocol [37].

The surface hopping trajectories are started from $S_{1}, S_{2}$ and $S_{3}$ (initial populations $0.06,0.73$ and 0.21 , respectively), with an average excitation energy of $4.74 \mathrm{eV}$. Trajectories in the $S_{3}$ state decay rapidly to $S_{2}$, and even more rapid is the transition from $S_{2}$ to $S_{1}$ (see Figure 5). In fact the $S_{2}$ minimum, readily accessible from the Franck-Condon region, coincides with a $S_{2} / S_{1}$ CI. From $S_{1}$ we have mainly transitions to $T_{2}$ and $T_{3}$, that follow two distinct pathways. In particular, $S_{1} \rightarrow T_{2}$ transitions occur mainly in a 

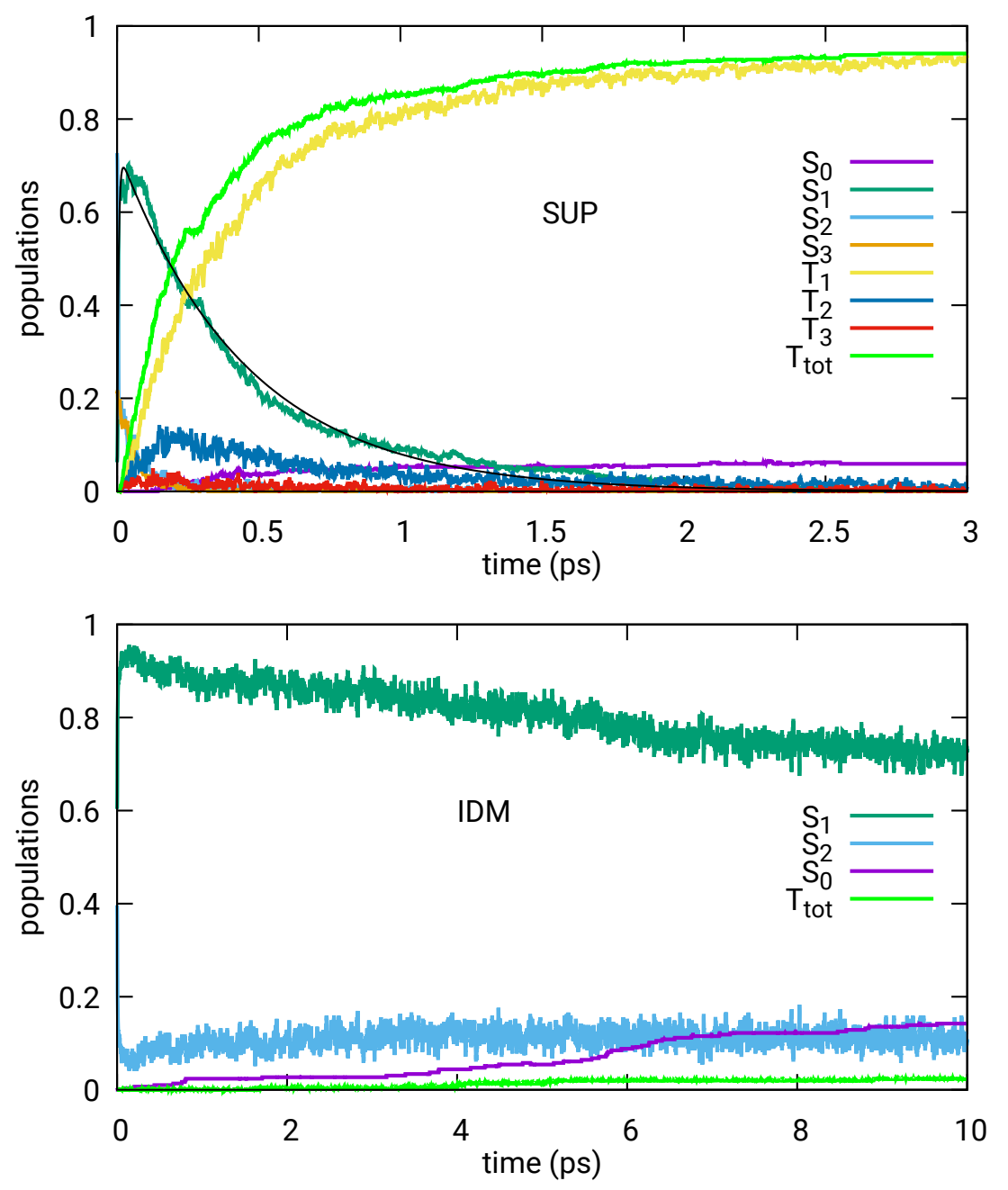

Figure 5: Time evolution of the populations of the spin-diabatic states for suprofen (upper panel) and indomethacin (lower panel). Note the different time scale for the two molecules (for suprofen, state populations are stable in the range $3-10$ ps). $T_{\text {tot }}$ is the total triplet population. The black curve is a fit of the suprofen $S_{1}$ population. 

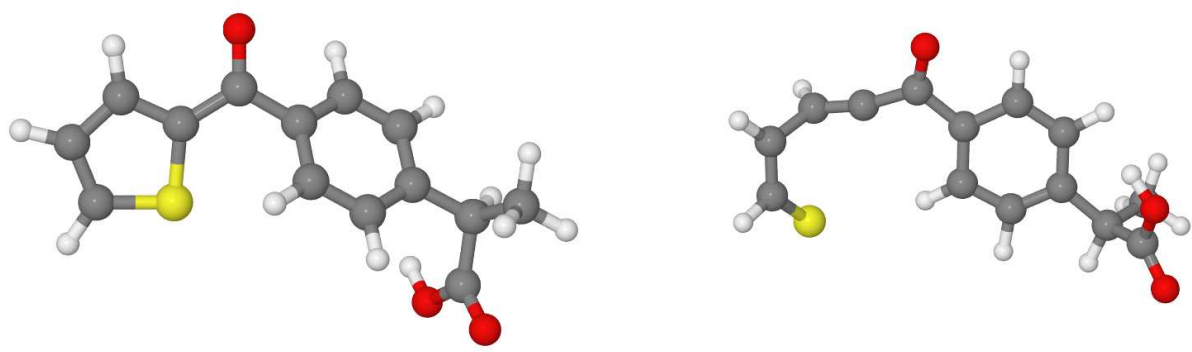

Figure 6: Ground state minimum of suprofen (left), and an exemplary geometry at which $S_{1} \rightarrow T_{2}$ and $S_{1} \rightarrow S_{0}$ transitions takes place (right).

degeneracy region where the bond between the sulfur and the carbon atom directly linked to the carbonyl group is broken, or at least is very stretched (see Figure 6). In fact, the average S-C distance at $S_{1}-T_{2}$ hops is $\mathrm{R}(\mathrm{S}-\mathrm{C})=2.70 \AA$. In all the trajectories but two (the total number of valid trajectories for SUP amount to 304, recall Table 1), the S-C bond is readily reconstructed after the hop. The transitions to $T_{3}$ occur instead in $S_{1} / T_{3}$ near degeneracy regions, where the thiophene ring is distorted (for example through the puckering at the $\mathrm{S}$ atom) although not opened. Once in the triplet manifold, very rapidly the $T_{1}$ state is reached, as the three triplets lie very close in energy to each other (recall the energies of the triplets at the Franck Condon region collected in Table 2).

The decay pattern reported above is in agreement with the character of the excited states. In fact, for $S_{1}$ and $T_{2}$ the excitation involves occupied orbitals mainly located on the thiophene moiety (the most important configurations for both states are $H \rightarrow L$ and $H \rightarrow L+1$, see Figure S1), while for $T_{3}$, which has a mixed character $H-2 \rightarrow L$ and $H-2 \rightarrow L+1$, the phenyl ring is mainly concerned. Accordingly, both $S_{1}$ and $T_{2}$ show a larger equilibrium S-C distance than $S_{0}(\mathrm{R}(\mathrm{S}-\mathrm{C})=1.75,1.69$ and $1.68 \AA$, respectively).

A minor but not negligible number of trajectories undergo $S_{1} \rightarrow S_{0}$ decay. After 10 ps the $S_{0}$ population is 0.06 , and the triplet quantum yield amounts to 0.94. Again, the $S_{1}-S_{0}$ transitions occur at large S-C distances, where $S_{0}, S_{1}, T_{1}$ and $T_{2}$ are almost degenerate (see Figure 6). The $S_{1}$ population can be fitted by a biexponential function, appropriate for a two-step first order irreversible decay kinetics

$$
P_{S_{1}}=w\left(e^{-t / \tau_{2}}-e^{-t / \tau_{1}}\right) .
$$

We obtain $w=0.74, \tau_{1}=0.005 \mathrm{ps}$ and $\tau_{2}=0.45 \mathrm{ps}$. In their transient absorption experiments with SUP in acetonitrile, Du et al. [8] registered a lifetime of $2.3 \mathrm{ps}$ for $S_{1}$. Moreover, in correspondence with the decay of the signal attributed to $S_{1}$, the rise of an absorption attributed to $T_{1}$ was apparent. This is in nice agreement with our results, although our lifetime $\tau_{2}$ for the $S_{1}$ decay is about five times smaller than the experimental value. Large amplitude motions, involved in the transitions from $S_{1}$ to triplets (for example, the opening and reforming of the thiophene cycle) are expected to be hindered in solution, slowing down the $S_{1}$ decay. The solvent may then explain, at least in part, the difference between our gas phase lifetimes and those obtained in the transient absorption experiments in solution.

\subsection{Indomethacin (IDM)}

The experimental absorption spectrum of IDM in water [9, 12] shows two peaks at $320 \mathrm{~nm}(3.87 \mathrm{eV})$ and at $267 \mathrm{~nm}(4.64 \mathrm{eV})$, to be compared with the vertical excitation energies $S_{0} \rightarrow S_{1}$ and $S_{0} \rightarrow S_{2}$ at 4.19 and $4.23 \mathrm{eV}$, shown in Table 2. The AM1 energies of these two absorptions are only $0.1 \mathrm{eV}$ below the vertical transitions predicted by the TD-CAMB3LYP/6-31+G(d,p) protocol, compare Table 2 and Table $\mathrm{S} 1$ of the Supporting Information. The fluorescence and phosphorescence spectra are also reported [9, 10], with maxima respectively at $380 \mathrm{~nm}(3.26 \mathrm{eV}$, in water $)$ and $475 \mathrm{~nm}(2.61 \mathrm{eV}$, in various organic solvents). The corresponding calculated $S_{1} \rightarrow S_{0}$ and $T_{1} \rightarrow S_{0}$ vertical transition energies are found at $3.52 \mathrm{eV}$ and $1.99 \mathrm{eV}$, respectively. Both $S_{1}$ and $S_{2}$ show some charge transfer character from the indole moiety to the 
benzoyl group. In fact, the Mulliken charge of the indole ring increases by about $0.15(0.18)$ going from $S_{0}$ to $S_{1}\left(S_{0}\right.$ to $\left.S_{2}\right)$.

At the beginning of the nonadiabatic dynamics simulations for IDM the population of $S_{1}$ and $S_{2}$ is 0.6 and 0.4 , respectively, with an average excitation energy of $4.15 \mathrm{eV}$. Due to the very small $S_{2}-S_{1}$ energy difference at the Franck-Condon geometry (only $0.04 \mathrm{eV}$ ), most of the population of $S_{2}$ is transferred to $S_{1}$ within the first $50 \mathrm{fs}$, which is by far the most populated state during the first 10 ps that last our simulations. Notice, however, that the trajectories keep hopping back and forth between $S_{1}$ and $S_{2}$, as it may be appreciated from the noisy character of $P_{S_{2}}$ and $P_{S_{1}}$ curves in Figure 5. This is due to the fact that the $S_{2}-S_{1}$ gap stays small at the geometries visited during the dynamics. In particular, the $S_{2}$ state is only $0.5 \mathrm{eV}$ above $S_{1}$ at the $S_{1}$ equilibrium geometry, while the $S_{2}$ minimum coincides with a $S_{2} / S_{1}$ CI. The average energy difference $S_{2}-S_{1}$ oscillates around about $0.5 \mathrm{eV}$ during the dynamics, which is an indication of the fact that most of the trajectories wander around the $S_{1}$ minimum region. It is from that region that the transitions $S_{1} \rightarrow S_{0}$ occur, leading to a $S_{0}$ population of 0.14 after 10 ps. A fit of the decay of $P_{S_{1}}+P_{S_{2}}$ delivers a lifetime of $55 \mathrm{ps}$. As already stated above, the accurate description of this kind of decay represents a particularly difficult task for surface hopping. Moreover, our simulation is limited to the very beginning of the IDM excited state dynamics (see Figure 5). For these reasons, our estimation of 55 ps for the singlet lifetime has to be taken as a rough approximation of the real value.

At the $S_{1}$ minimum, the energy difference $S_{1}-T_{3}$ and $S_{1}-T_{2}$ is only 0.11 and $0.35 \mathrm{eV}$, respectively. A small number of trajectories (7) undergo intersystem crossing from $S_{1}$ to the nearby triplets $T_{2}$ or $T_{3}$, leading to a triplet population of 0.02 after $10 \mathrm{ps}$. It is of course not possible to extract a triplet quantum yield from these data. However, they are in agreement with the experimental observation of phosphorescence for IDM.

\section{Concluding remarks}

We have undertaken a computational investigation of the excited state dynamics of some NSAIDs, using the surface hopping approach and including the spin-orbit interaction to account for intersystem crossing processes. Although PES and wavefunctions were obtained in a semiempirical framework retaining the standard AM1 parameters, overall our FOMO-CI energies showed a quite good agreement with the experimental spectroscopic data available.

ASP and IBU, which share the benzene group as chromophore, show a relatively fast decay to the ground state, with very little or no population of the triplet states, with only a modest solvent slowdown for $\mathrm{ASP}_{a q}$. The decay is especially fast in IBU, which has a singlet lifetime of only $\sim 2$ ps. Transitions to $S_{0}$ are triggered by distortions of the aromatic ring (especially for IBU), in particular involving the carbon atoms linked to the substituents.

CAR and SUP show ultrafast decay to $T_{1}$, with time constants for the rise of the triplet population of 1.04 and $0.31 \mathrm{ps}$, respectively, and triplet quantum yields close to 1 . However, the decay pattern is quite different: for CAR, $S_{1}$ and $S_{2}$ are very close in energy to $T_{3}$ and $T_{4}$, both at the Franck-Condon point and at the $S_{1}$ and $S_{2}$ minima, and the singlet/triplet transitions take place without relevant distortions of the polycyclic conjugated system. For SUP, transitions to triplets involve severe deformations of the thiophene moiety.

An experimental time-resolved absorption study of excited state dynamics of SUP in acetonitrile by Du et al. is available in the literature [8]. They obtain a decrease of the $S_{1}$ signal simultaneous to the increase of the $T_{1}$ signal, which is only in qualitative agreement with our results, as their time constants for the singlet decay is about five times larger than ours ( 2.3 versus $0.45 \mathrm{ps}$, respectively). However, it is our opinion that the solvent could play a role in slowing down the intersystem crossing rate, especially considering the large amplitude motion we found involved in this process for suprofen.

A still different behavior is shown by IDM, where the $S_{1}$ state show a very slow decay, with a dynamics which is far from being completed after the first 10 ps. Among the NSAIDs considered in the present work, clearly the IDM dynamics is the least suitable for a surface hopping investigation.

The molecular dynamics results from this study, together with the information inferred from the static works of other groups on this family of NSAIDS, reveal the complexity of the new descriptors to be designed 
to capture the stability or reactivity of drugs exposed to light of different wavelengths and the deficiency of simple models such as for instance the one based on the magnitude of HOMO-LUMO gap. In fact, the traditional HOMO-LUMO gap model fails to predict the photoreactivity/photostability of 3 out of the 5 systems considered in this work by classifying IDM $(8.15 \mathrm{eV}), \mathrm{CAR}(8.14 \mathrm{eV})$, and SUP (8.81 eV) as unlikely phototoxic systems attending to their H-L gaps which exceed the threshold value of $8.1 \mathrm{eV}$ [38] (H-L gaps provided within parenthesis correspond to the most stable conformers calculated at AM1 level of theory).

This and other works bring forward the importance of the topography of the potential energy surface far away of the Franck-Condon region, revealing crucial information on the inherent stability of these chromophores connected to the existence of excited state minima that could potentially trap the population in the excited state or the accessibility of decaying funnels or for the transfer of population to manifolds of other multiplicities. Interestingly, in the case where several decay routes are competitive, time resolved approaches allow determining their relative importance in the global decay mechanisms. Finally, our study also discloses the importance of accounting for solvent solute interactions, which as shown here might importantly tune the decay mechanisms and lifetimes.

\section{Acknowledgments}

N. A. thanks the Marie Curie Actions, within the Innovative Training Network-European Joint Doctorate in Theoretical Chemistry and Computational Modelling TCCM-ITN-EJD-642294, for financial support. G.G. acknowledges funding from the University of Pisa, PRA_2017_28. I.C. gratefully acknowledges the "Ramón y Cajal" program and financial support from the Project CTQ2015-63997-C2 of the Ministerio de Economía y Competitividad of Spain.

\section{Appendix A.}

Transition probabilities in surface hopping are usually obtained according to Tully's "fewest switches" prescription [24, 39]. It has been noted [40] that the fewest switches algorithm used in local diabatization (LD) schemes [25, 26, 41] is physically not well grounded and subject to numerical instabilities, expecially in the presence of many weakly interacting states. In view of these difficulties, we propose here an alternative procedure.

For a given surface hopping trajectory, the electronic wavefunction at time $t$ is expanded on the adiabatic basis $|\varphi\rangle$

$$
\Psi_{e l}(t)=\sum_{j} C_{j}(t)\left|\varphi_{j}(t)\right\rangle
$$

The population of state $\left|\varphi_{j}\right\rangle$ is then $P_{j}(t)=\left|C_{j}(t)\right|^{2}$. In the LD scheme, the integration of the timedependent Schrödinger equation for the electrons is performed in an alternative "locally diabatic" electronic basis $|\boldsymbol{\eta}\rangle$, spanning the same subspace as the adiabatic basis $|\boldsymbol{\varphi}\rangle$, and defined so as to be (approximately) constant in the integration time step $\Delta t$

$$
\begin{aligned}
|\boldsymbol{\eta}(0)\rangle & =|\boldsymbol{\varphi}(0)\rangle \\
|\boldsymbol{\eta}(\Delta t)\rangle & \equiv|\boldsymbol{\eta}(0)\rangle \simeq|\boldsymbol{\varphi}(\Delta t)\rangle \mathbf{T}^{\dagger}
\end{aligned}
$$

Here we set $t=0$ at the beginning of the time step. The unitary matrix $\mathbf{T}(\Delta t)$ is obtained by Löwdin orthonormalization of the overlap matrix $\langle\varphi(0) \mid \varphi(\Delta t)\rangle$ between the adiabatic wavefunctions at the beginning and at the end of the time step.

Let $\left|\varphi_{k}\right\rangle$ be the current state (i.e. the state on which PES the trajectory is running). We label $W_{k}$ the total transition probability from state $k$. In agreement with the fewest switches algorithm, $W_{k}$ is given by

$$
W_{k}=\max \left\{0, \frac{P_{k}(0)-P_{k}(\Delta t)}{P_{k}(0)}\right\}
$$


The problem is how to partition $W_{k}$ among the adiabatic states $j \neq k$ in order to find the transition probabilities $T_{k \rightarrow j}$ from the current state $k$ to the other adiabatic states $j$. Notice that in a two state case there is nothing to partition, the transition probability $T_{k \rightarrow j}$ being just equal to $W_{k}$. We assume then in the following that the number of states is larger than 2.

For $W_{k}=0$ we set $T_{k \rightarrow j}=0$. Assuming $W_{k}>0$, we want to define $T_{k \rightarrow j}>0$ such that $\sum_{j \neq k} T_{k \rightarrow j}=W_{k}$. To this aim, we notice that $T_{k \rightarrow j}$, according to the fewest switches algorithm, has to depend on the population increment of state $j$ within the time step $\Delta t$ and on the coupling between states $k$ and $j$, which is in turn proportional to the overlap $\left\langle\varphi_{k}(0) \mid \varphi_{j}(\Delta t)\right\rangle$. We introduce then the following quantities

$$
x_{k j}=\frac{\left|\mathbf{T}_{k j}\right| \sqrt{\Delta P_{j}}}{S_{k}} \quad \text { with } \quad S_{k}=\sum_{j \neq k}\left|\mathbf{T}_{k j}\right| \sqrt{\Delta P_{j}}
$$

where $\Delta P_{j}=\max \left\{0, P_{j}(\Delta t)-P_{j}(0)\right\}$ is the population increment for state $j$. The non negative quantities $x_{k j}$ define the partition, so that the fewest switches transition probability is given by

$$
T_{k \rightarrow j}=x_{k j} W_{k} .
$$

The peculiar functional form of equation A.5 for $S_{k}$ has been chosen considering that, according to the LD propagation algorithm of ref. [25], the $P_{j}(\Delta t)$ probabilities are proportional to the square of the $\mathbf{T}$ matrix elements. A good indication of the fact that the partition induced by the factors $x_{k j}$ is correct would be that the normalization term $S_{k}$ is equal to the decrement of the current state population. Actually, this can be easily shown for a system that wanders in a region of weak coupling, where $P_{k}(0) \simeq 1$ and $P_{j}(0) \simeq 0$ for $j \neq k$ (as it would be enforced by any algorithm aimed at introducing quantum decoherence effects). Therefore, according to equation 16 of ref. [25], $P_{j}(\Delta t) \simeq\left|\mathbf{T}_{k j}\right|^{2}$. Moreover, $W_{k} P_{k}(0) \simeq W_{k} \simeq 1-\left|\mathbf{T}_{k k}\right|$. Hence, as $\mathbf{T}$ is unitary, we have $S_{k} \approx W_{k} P_{k}(0)$, which is what we wanted to prove.

Good properties of the algorithm outlined above are: (i) easily implemented and based on quantities already available in a LD computation; (ii) the partition proposed is physically sound and not subject to numerical instabilities; (iii) the sum of the $T_{k \rightarrow j}$ is equal to $W_{k}$ by construction (at variance with a previously proposed improvement [40] of the original fewest switches algorithm [25] for LD).

\section{References}

[1] V. Lhiaubet-Vallet, M. A. Miranda, Phototoxicity of drugs, in: F. G. A. Griesbeck M. Oelgemöller (Ed.), CRC Handbook of Organic Photochemistry and Photobiology, CRC Press, Boca Raton, 2012, pp. 1541-1555.

[2] K. A. K. Musa, L. A. Eriksson, Theoretical investigation of NSAID photodegradation mechanisms, in: C. F. Matta (Ed.), Quantum Biochemistry, Wiley, Weinheim, 2010, pp. 805-834.

[3] K. Florey, Aspirin, Analytical Profiles of Drug Substances 8 (1979) 1-46. doi:10.1016/S0099-5428(08)60112-4

[4] D. Budac, P. Wan, Photodecarboxylation: mechanism and synthetic utility, J. Photochem. Photobiol. A: Chemistry 67 (1992) 135-166. doi:10.1016/1010-6030(92)85224-I

[5] D. Vione, P. R. Maddigapu, E. DeLaurentiis, M. Minella, M. Pazzi, V. Maurino, C. Minero, S. Kouras, C. Richard, Modelling the photochemical fate of ibuprofen in surface waters, Water Research 45 (2011) $6725-6736$. doi:10.1016/j.watres.2011.10.014

[6] F. Bosca, S. Encinas, P. F. Heelis, M. A. Miranda, Photophysical and photochemical characterization of a photosensitizing drug: A combined steady state photolysis and laser flash photolysis study on carprofen, Chem. Res. Toxicol. 10 (1997) 820-827. doi:10.1021/tx9700376.

[7] M.-T. Sheu, H.-O. Ho, P.-Y. Wang, Y.-B. Liou, A.-B. Wu, Photolysis of NSAIDs. I. Photodegradation products of carprofen determined by LC-ESI-MS, J. Chrom. Sci. 41 (2003) 200-204. doi:10.1093/chromsci/41.4.200

[8] L. Du, R. Zhu, J. Xue, Y. Du, D. L. Phillips, Time-resolved spectroscopic and density functional theory investigation of the photochemistry of suprofen, J. Raman Spectrosc. 46 (2015) 117-125. doi:\{10.1002/jrs.4598\}.

[9] B. Maity, A. Chatterjee, S. A. Ahmed, D. Seth, Interaction of the nonsteroidal anti-inflammatory drug indomethacin with micelles and its release, J. Phys. Chem. B 119 (2015) 3776-3785. doi:10.1021/acs.jpcb.5b00467.

[10] R. Dabestani, R. H. Sik, D. G. Davis, G. Dubay, C. F. Chignell, Spectroscopic studies of cutaneous photosensitizing agents. XVIII. Indomethacin, Photochem. Photobiol. 58 (1993) 367-373. doi:10.1111/j.1751-1097.1993.tb09576.x

[11] D. E. Moore, P. P. Chappuis, A comparative study of the photochemistry of the non-steroidal antiinflammatory drugs, naproxen, benoxaprofen and indomethacin, Photochem. Photobiol. $47 \quad 173-180$. doi:10.1111/j.1751-1097.1988.tb02710.x

[12] A. Ghatak, P. C. Mandal, M. Sarkar, Indomethacin: A nsaid sensitive to micro heterogeneity in alcohol-water mixtures, Chem. Phys. Lett. 460 (2008) 521-524. doi:10.1016/j.cplett.2008.06.056 
[13] A. C. Weedon, D. F. Wong, The photochemistry of indomethacin, J. Photochem. Photobiol. A: Chemistry 61 (1991) 27-33. doi:10.1016/1010-6030(91)85071-N

[14] M. M. Miotke, M. Józefowicz, Solvatochromism of antiinflammatory drug - naproxen sodium, J. Mol. Liq. 230 (2017) 129-136. doi:10.1016/j.molliq.2016.12.094

[15] P. Govindasamy, S. Gunasekaran, S. Srinivasan, Molecular geometry, conformational, vibrational spectroscopic, molecular orbital and Mulliken charge analysis of 2-acetoxybenzoic acid, Spectrochim. Acta Part A 130 (2014) $329-336$. doi:10.1016/j.saa.2014.03.056

[16] Y. Ye, G. Tang, Y. Han, L. F. Culnane, J. Zhao, Y. Zhang, Dft studies on the vibrational and electronic spectra of acetylsalicylic acid, Optics and Spectroscopy 120 (2016) 680-689. doi:10.1134/S0030400X1605026X

[17] K. A. K. Musa, V. R. Palwai, L. A. Eriksson, New nonsteroidal anti-inflammatory molecules with reduced photodegradation side effects and enhanced COX-2 selectivity, Int. J. Quantum Chem. 111 (2011) 1184-1195. doi:10.1002/qua.22649

[18] K. A. K. Musa, L. A. Eriksson, Theoretical study of ibuprofen phototoxicity, J. Phys. Chem. B 111 (2007) $13345-13352$. doi:10.1021/jp076553e

[19] A. V. Gubskaya, Quantum-chemical descriptors in QSAR/QSPR modeling: achievements, perspectives and trends, in: C. F. Matta (Ed.), Quantum Biochemistry, Wiley, Weinheim, 2010, pp. 805-834.

[20] G. Granucci, A. Toniolo, Molecular gradients for semiempirical CI wavefunctions with floating occupation molecular orbitals, Chem. Phys. Lett. 325 (2000) 79-85. doi:10.1016/S0009-2614(00)00691-6.

[21] M. Persico, G. Granucci, An overview of nonadiabatic dynamics simulations methods, with focus on the direct approach versus the fitting of potential energy surfaces, Theor. Chem. Acc. 133 (2014) 1526. doi:10.1007/s00214-014-1526-1

[22] G. Granucci, M. Persico, Gradients for Configuration Interaction energies with spin-orbit coupling in a semiempirical framework, J. Comput. Chem. 32 (2011) 2690-2696. doi:10.1002/jcc.21850

[23] L. Martínez-Fernández, I. Corral, G. Granucci, M. Persico, Competing ultrafast intersystem crossing and internal conversion: A time resolved picture for the deactivation of 6-thioguanine, Chem. Sci. 5 (2014) 1336-1347. doi:10.1039/C3SC52856A

[24] J. C. Tully, Molecular dynamics with electronic transitions, J. Chem. Phys. 93 (1990) 1061-1071. doi:10.1063/1.459170

[25] G. Granucci, M. Persico, A. Toniolo, Direct semiclassical simulation of photochemical processes with semiempirical wavefunctions, J. Chem. Phys. 114 (2001) 10608. doi:10.1063/1.1376633

[26] R. Crespo-Otero, M. Barbatti, Recent advances and perspectives on nonadiabatic mixed quantum-classical dynamics, Chem. Rev. 118 (2018) 7026-7068. doi:10.1021/acs.chemrev.7b00577

[27] F. Plasser, G. Granucci, J. Pittner, M. Barbatti, M. Persico, H. Lischka, Surface hopping dynamics using a locally diabatic formalism: Charge transfer in the ethylene dimer cation and excited state dynamics in the 2-pyridone dimer, J. Chem. Phys. 137 (2012) 22A514. doi:10.1063/1.4738960

[28] G. Granucci, M. Persico, A. Zoccante, Including quantum decoherence in surface hopping, J. Chem. Phys. 133 (2010) 134111. doi:10.1063/1.3489004

[29] T. Cusati, G. Granucci, M. Persico, Photodynamics and time-resolved fluorescence of azobenzene in solution: A mixed quantum-classical simulation, J. Am. Chem. Soc. 133 (2011) 5109-5123. doi:10.1021/ja1113529

[30] G. Bussi, M. Parrinello, Stochastic thermostats: comparison of local and global schemes, Comp. Phys. Commun. 179 (2008) 26-29. doi:10.1016/j.cpc.2008.01.006

[31] J. J. P. Stewart, MOPAC2000; Fujitsu Limited: Tokyo, Japan (1999).

[32] J. W. Ponder, TINKER - Software Tools for Molecular Design, Version 6.3 (2014).

[33] M. O. Iwunze, Absorptiometric determination of acetylsalicylic acid in aqueous ethanolic solution, Analyt. Lett. 41 (2008) 2944-2953. doi:10.1018/00032710802440574

[34] V. Cantatore, G. Granucci, M. Persico, Simulation of the $\pi \rightarrow \pi^{*}$ photodynamics of azobenzene: Decoherence and solvent effects, Comput. Theor. Chem. 1040-1041 (2014) 126-135. doi:10.1016/j.comptc.2014.02.011.

[35] F. Yuan, C. Hu, X. Hu, J. Qu, M. Yang, Degradation of selected pharmaceuticals in aqueous solution with UV and $\mathrm{UV} / \mathrm{H}_{2} \mathrm{O}_{2}$, Water Research 43 (2009) 1766-1774. doi:10.1016/j.watres.2009.01.008.

[36] J. Moser, F. Boscá, W. W. Lovell, J. V. Castell, M. A. Miranda, A. Hye, Photobinding of carprofen to protein, J. Photochem. Photobiol. B: Biol. 58 (2000) 13-19. doi:10.1016/S1011-1344(00)00115-9

[37] K. A. K. Musa, L. A. Eriksson, Photodegradation mechanism of nonsteroidal anti-inflammatory drugs containing thiophene moieties: Suprofen and tiaprofenic acid, J. Phys. Chem. B 113 (2009) 11306-11313. doi:10.1021/jp904171p

[38] S. Peukert, J. Nunez, F. He, M. Dai, N. Yusuff, A. DiPesa, K. Miller-Moslin, R. Karki, B. Lagu, C. Harwell, Y. Zhang, D. Bauer, J. F. Kelleher, W. Egan, A method for estimating the risk of drug-induced phototoxicity and its application to smoothened inhibitors, Med. Chem. Commun. 2 (2011) 973-976. doi:10.1039/C1MD00144B.

[39] S. Hammes-Schiffer, J. C. Tully, Proton transfer in solution: Molecular dynamics with quantum transitions, J. Chem. Phys. 101 (1994) 4657-4667. doi:10.1063/1.467455

[40] J. J. Bajo, G. Granucci, M. Persico, Interplay of radiative and nonradiative transitions in surface hopping with radiationmolecule interactions, J. Chem. Phys. 140 (2014) 044113. doi:10.1063/1.4862738

[41] S. Mai, P. Marquetand, L. González, Nonadiabatic dynamics: The SHARC approach, WIREs Comput. Mol. Sci doi: $10.1002 /$ wcms. 1370 